\title{
Exploring Partonic Structure of Hadrons Using ab initio Lattice QCD Calculations
}

\author{
Yan-Qing $\mathrm{Ma}^{1,2,3, *}$ and Jian-Wei Qiu ${ }^{4, \dagger}$ \\ ${ }^{1}$ School of Physics and State Key Laboratory of Nuclear Physics and Technology, Peking University, Beijing 100871, China \\ ${ }^{2}$ Center for High Energy physics, Peking University, Beijing 100871, China \\ ${ }^{3}$ Collaborative Innovation Center of Quantum Matter, Beijing 100871, China \\ ${ }^{4}$ Theory Center, Jefferson Lab, 12000 Jefferson Avenue, Newport News, Virginia 23606, USA
}

(Received 24 September 2017; published 10 January 2018)

\begin{abstract}
Following our previous proposal, we construct a class of good "lattice cross sections" (LCSs), from which we can study the partonic structure of hadrons from ab initio lattice QCD calculations. These good LCSs, on the one hand, can be calculated directly in lattice QCD, and on the other hand, can be factorized into parton distribution functions (PDFs) with calculable coefficients, in the same way as QCD factorization for factorizable hadronic cross sections. PDFs could be extracted from QCD global analysis of the lattice QCD generated data of LCSs. We also show that the proposed functions for lattice QCD calculation of PDFs in the literature are special cases of these good LCSs.
\end{abstract}

DOI: 10.1103/PhysRevLett.120.022003

Introduction.-Parton distributions functions (PDFs), interpreted as probability distributions to find an active parton from a colliding hadron to carry the $x$ fraction of the parent hadron momentum, are very important noperturbative quantities. They connect hadronic cross sections with a large momentum transfer to perturbatively calculable partonic dynamics, so that we can interpret high energy scattering data and make predictions for future observables [1-3]. PDFs have been extracted by performing global analysis of high energy scattering data in the framework of QCD factorization [4-8]. Since PDFs are well defined in $\mathrm{QCD}$, it is not only very natural, but also critically important to ask and verify if PDFs could be derived from the first-principles calculations in lattice QCD (LQCD). However, a direct calculation of PDFs in LQCD is challenging due to the fact that PDFs are defined in terms of operators with a Minkowski time dependence, while LQCD calculations are done with a Euclidean time.

Moments of PDFs, given by matrix elements of local operators, can be, in principle, calculated in LQCD. However, in practice, calculations are limited to the lowest three moments [9] because of power-divergent mixing between twist- 2 operators. In Ref. [10], Ji introduced a set of quasi-PDFs, which are defined with the same operators defining PDFs except the active parton fields are not located on the light cone, but on a spatial axis (along the $z$ or " 3 " direction) with no time separation, and could be

Published by the American Physical Society under the terms of the Creative Commons Attribution 4.0 International license. Further distribution of this work must maintain attribution to the author(s) and the published article's title, journal citation, and DOI. Funded by SCOAP ${ }^{3}$. calculated in LQCD [11-15]. It was also suggested that quasi-PDFs could approach to corresponding PDFs when the hadron momentum $P_{3}$ goes to infinity $[10,16,17]$. In Ref. [1], it was demonstrated that if quasi-PDFs are multiplicatively renormalizable, they could be related to PDFs by an all-order QCD factorization at a finite $P_{3}$. Major progress has been made in understanding the complexity of ultraviolet (UV) divergences of quasi-PDFs [18-28]. Meanwhile, various different methods have been introduced to study hadron structures using LQCD calculations, including the pseudo-PDFs approach [29] and the "OPE without OPE" approach [30], as well as the approach to extract hadron structure functions from hadronic tensors that are converted from lattice QCD calculated ones in Euclidean space to Minkowski space by using the inverse Laplace transform [31-33]. For light-cone distribution amplitudes, which are much simpler than PDFs, a coordinate-space method was also employed [34-36].

In Ref. [1], we proposed a factorization-based program to extract PDFs and other parton correlation functions by using QCD global analysis of "data" generated by LQCD calculations of "lattice cross sections" (LCSs), which are defined as factorizable and "time-independent" hadronic matrix elements (defined by equal-time operators or with the time properly integrated). More precisely, a good LCS for extracting PDFs should have the following properties [1]: (i) is calculable in LQCD with a Euclidean time, (ii) has a well-defined continuum limit as the lattice spacing $a \rightarrow 0$, and (iii) has the same and factorizable logarithmic collinear (CO) divergences as PDFs. It is the last property that enables us to relate good LCSs to PDFs, just like how hadronic cross sections are related to PDFs in terms of QCD factorization. We also argued that quasi-quark distribution is an example of good LCSs. 
In this Letter, we further strengthen our proposal by constructing a class of good LCSs, with which we could build up a comprehensive program to explore the partonic structure of various hadrons with many LQCD calculable observables. We demonstrate that these LCSs have the three required properties listed above for being good LCSs. We also comment that quasi-PDFs proposed in Ref. [10], pseudo-PDFs used in Ref. [29], and the matrix element used in Ref. [30] are special cases of these good LCSs. The proposed method of using good LCSs could be the most general way to extract PDFs from LQCD calculations.

Hadronic matrix elements in coordinate space.-We consider single-hadron matrix elements of renormalized nonlocal operators $\mathcal{O}_{n}(\xi)$,

$$
\sigma_{n}\left(\omega, \xi^{2}, P^{2}\right)=\left\langle P\left|T\left\{\mathcal{O}_{n}(\xi)\right\}\right| P\right\rangle,
$$

where the subscript $n$ is a label for different operators, $T$ stands for time ordering, $P$ is the hadron momentum, $\xi$ with $\xi^{2} \neq 0$ is the largest separation of all fields in the operator $\mathcal{O}_{n}$, the Lorentz scalar $\omega \equiv P \cdot \xi$, and renormalization scale for $\mathcal{O}_{n}(\xi)$ is suppressed.

One choice for $\mathcal{O}_{n}(\xi)$ is the dimension-2 operators for correlations of two currents with a separation $\xi$,

$$
\mathcal{O}_{j_{1} j_{2}}(\xi) \equiv \xi^{d_{j_{1}}+d_{j_{2}}-2} Z_{j_{1}} Z_{j_{2}} j_{1}(\xi) j_{2}(0)
$$

where $d_{j}$ and $Z_{j}$ are the dimension and renormalization constant of the current $j$, respectively, and the overall dimensional factor is introduced so that the matrix elements in Eq. (1) are dimensionless with our normalization, $\left\langle P \mid P^{\prime}\right\rangle=\left(2 E_{P}\right)(2 \pi)^{3} \delta^{3}\left(P-P^{\prime}\right)$. With the scalar and vector currents, for example, we could have

$$
\begin{aligned}
\mathcal{O}_{S}(\xi) & =\xi^{4} Z_{S}^{2}\left[\bar{\psi}_{q} \psi_{q}\right](\xi)\left[\bar{\psi}_{q} \psi_{q}\right](0), \\
\mathcal{O}_{V}(\xi) & =\xi^{2} Z_{V}^{2}\left[\bar{\psi}_{q} \not{\xi} \psi_{q}\right](\xi)\left[\bar{\psi}_{q} \xi_{q}\right](0), \\
\mathcal{O}_{\tilde{V}}(\xi) & =-\frac{\xi^{4}}{2} Z_{V}^{2}\left[\bar{\psi}_{q} \gamma_{\nu} \psi_{q}\right](\xi)\left[\bar{\psi}_{q} \gamma^{\nu} \psi_{q}\right](0), \\
\mathcal{O}_{V^{\prime}}(\xi) & =\xi^{2} Z_{V^{\prime}}^{2}\left[\bar{\psi}_{q} \not{\xi} \psi_{q^{\prime}}\right](\xi)\left[\bar{\psi}_{q^{\prime}} \not{\xi} \psi_{q}\right](0), \ldots,
\end{aligned}
$$

where $\xi^{4} \equiv\left(\xi^{2}\right)^{2}, q=u, d, s, \ldots$ stands for a quark with a definite flavor and $q^{\prime}$ for a quark with a different flavor, the subscripts, $S, V$, and $V^{\prime}$ refers to scalar, vector and flavorchanging vector currents, respectively, and "..." indicates for other possible combinations of two currents including the gluonic current, e.g., $j_{\mu \nu} \propto F_{\mu \rho} F_{\nu}^{\rho}$. Matrix elements constructed from operators in Eq. (3) satisfy the relation

$$
\sigma_{n}^{*}\left(\omega, \xi^{2}, P^{2}\right)=\sigma_{n}\left(-\omega, \xi^{2}, P^{2}\right) .
$$

Instead of the correlation of two currents, the nonlocal operator in Eq. (1) could also be made of the correlation of gauge dependent field operators with proper gauge link(s), e.g.,

$$
\mathcal{O}_{q}(\xi)=Z_{q}\left(\xi^{2}\right) \bar{\psi}_{q}(\xi) \not{\not} \Phi(\xi, 0) \psi_{q}(0),
$$

where $\Phi(\xi, 0)=\mathcal{P} e^{-i g \int_{0}^{1} \xi \cdot A(\lambda \xi) d \lambda}$ is the path ordered gauge link, $Z_{q}\left(\xi^{2}\right)$ is the renormalization constant of this operator, depending on $\xi^{2}$ [27], and the matrix element constructed from which satisfies the relation

$$
\sigma_{n}^{*}\left(\omega, \xi^{2}, P^{2}\right)=-\sigma_{n}\left(-\omega, \xi^{2}, P^{2}\right) .
$$

Besides scalar operators constructed above, we can also construct vector or tensor operators, e.g.,

$$
\mathcal{O}_{\mu \nu}(\xi)=\xi^{4} Z_{V}^{2}\left[\bar{\psi}_{q} \gamma_{\mu} \psi_{q}\right](\xi)\left[\bar{\psi}_{q} \gamma_{\nu} \psi_{q}\right](0) .
$$

To simply the discussion, we will consider only scalar operators in the following, although tensor operators can be studied the same way.

Factorization.-We show that $\sigma_{n}$ defined in Eq. (1) could be factorized into PDFs with perturbatively calculable coefficients so long as $\xi^{2}$ is sufficiently small,

$$
\begin{aligned}
\sigma_{n}\left(\omega, \xi^{2}, P^{2}\right)= & \sum_{a} \int_{-1}^{1} \frac{d x}{x} f_{a}\left(x, \mu^{2}\right) K_{n}^{a}\left(x \omega, \xi^{2}, x^{2} P^{2}, \mu^{2}\right) \\
& +O\left(\xi^{2} \Lambda_{\mathrm{QCD}}^{2}\right),
\end{aligned}
$$

where $\mu$ is the factorization scale, $K_{n}^{a}$ are perturbatively calculable hard coefficients, and $f_{a}$ is the PDF of flavor $a=q, g$ with antiquark PDFs expressed by quark PDFs using the relation $f_{\bar{a}}\left(x, \mu^{2}\right)=-f_{a}\left(-x, \mu^{2}\right)$.

Let $\xi^{2}$ be small but not vanishing, and applying operator product expansion (OPE) to the nonlocal operator $\mathcal{O}_{n}(\xi)$ in Eq. (1) [37], we have

$$
\begin{aligned}
\sigma_{n}\left(\omega, \xi^{2}, P^{2}\right)= & \sum_{J=0} \sum_{a} W_{n}^{(J, a)}\left(\xi^{2}, \mu^{2}\right) \xi^{\nu_{1}} \ldots \xi^{\nu_{J}} \\
& \times\left\langle P\left|\mathcal{O}_{\nu_{1} \cdots \nu_{J}}^{(J, a)}\left(\mu^{2}\right)\right| P\right\rangle,
\end{aligned}
$$

where $\mu$ is the renormalization scale. The $\mathcal{O}_{\nu_{1} \cdots \nu_{J}}^{(J, a)}\left(\mu^{2}\right)$ is a local, symmetric, and traceless operator of $\operatorname{spin} J$ with $a$ labeling different operators of the same spin, and

$$
\left\langle P\left|\mathcal{O}_{\nu_{1} \ldots \nu_{J}}^{(J, a)}\left(\mu^{2}\right)\right| P\right\rangle=2 A^{(J, a)}\left(\mu^{2}\right)\left(P_{\nu_{1}} \ldots P_{\nu_{J}}-\text { traces }\right),
$$

where the scalar quantity $A^{(J, a)}\left(\mu^{2}\right)=\left\langle P\left|\mathcal{O}^{(J, a)}\left(\mu^{2}\right)\right| P\right\rangle$ is the reduced matrix element. Substituting Eq. (10) into Eq. (9), we have

$$
\begin{aligned}
& \sigma_{n}\left(\omega, \xi^{2}, P^{2}\right) \\
& \quad=\sum_{J=0} \sum_{a} W_{n}^{(J, a)}\left(\xi^{2}, \mu^{2}\right) 2 A^{(J, a)}\left(\mu^{2}\right) \Sigma_{J}\left(\omega, P^{2} \xi^{2}\right),
\end{aligned}
$$

where 


$$
\begin{aligned}
\Sigma_{J}\left(\omega, P^{2} \xi^{2}\right) & \equiv \xi^{\nu_{1}} \ldots \xi^{\nu_{J}}\left(P_{\nu_{1}} \ldots P_{\nu_{J}}-\text { traces }\right) \\
& =\sum_{i=0}^{[J / 2]} C_{J-i}^{i}(\omega)^{J-2 i}\left(-P^{2} \xi^{2} / 4\right)^{i},
\end{aligned}
$$

where $C$ is the binomial function and $[J / 2]$ is the greatest integer less than or equal to $J / 2$. Up to now, no approximation has been made in deriving Eq. (11).

Since a higher dimensional matrix element is relatively smaller by powers of $\Lambda_{\mathrm{QCD}}^{2} \xi^{2}$ when two reduced matrix elements are compared, for the following discussion, we ignore this power suppressed correction to keep only terms with the lowest dimensional operators, which corresponds to keep the twist-2 operators in QCD [37]. Reduced matrix elements of these twist-2 operators can be expressed as moments of PDFs,

$$
A^{(J, a)}\left(\mu^{2}\right)=\frac{1}{S_{a}} \int_{-1}^{1} d x x^{J-1} f_{a}\left(x, \mu^{2}\right),
$$

where symmetry factor $S_{a}=1,2$ for $a=q, g$, respectively, and $J \geq 1$ because there is no scalar twist-2 operator. By substituting Eq. (13) into Eq. (11), and comparing it with Eq. (8) with the $K_{n}^{a}$ expanded in a power series of $\omega$, we prove that $\sigma_{n}$ in Eq. (11) has the factorized form in Eq. (8) with

$$
K_{n}^{a}=\sum_{J=1} \frac{2}{S_{a}} W_{n}^{(J, a)}\left(\xi^{2}, \mu^{2}\right) \Sigma_{J}\left(x \omega, x^{2} P^{2} \xi^{2}\right) .
$$

Note, however, that our proof is valid only when $|\omega| \ll 1$ and $\left|P^{2} \xi^{2}\right| \ll 1$ because OPE works only in the region where all components of $\xi$ go to zero uniformly but with all other variables fixed. That is, we need to further extend our proof to other regions, especially when $\omega$ is finite.

The validity of OPE guarantees that $\sigma_{n}$ is an analytic function of $\omega$ in the neighborhood of $\omega=0$, and its Taylor series of $\omega$ around $\omega=0$ is defined by Eqs. (9)-(14). If we fix $\xi$ to be at short distance while we increase $\omega$ by adjusting external momentum $P$, we cannot introduce any new perturbative divergence. That is, $\sigma_{n}$ remains to be analytic as $\omega$ becomes larger, and only possible singularity is at $\omega=\infty$. Similarly, for fixed $\xi, \sigma_{n}$ is an analytic function of $P^{2} \xi^{2}$ except for the point of infinity. Therefore, the factorization in Eq. (8), defined by a Taylor series of $\omega$ and $P^{2} \xi^{2}$, holds for any finite value of $\omega$ and $P^{2} \xi^{2}$ with the correction up to $O\left(\xi^{2} \Lambda_{\mathrm{QCD}}^{2}\right)$.

Note that the analytic behavior of $\sigma_{n}$ discussed above could be significantly different when it is Fourier transformed into momentum space, i.e.,

$$
\tilde{\sigma}_{n}\left(\tilde{\omega}, q^{2}, P^{2}\right) \equiv \int \frac{d^{4} \xi}{\xi^{4}} e^{i q \cdot \xi} \sigma_{n}\left(P \cdot \xi, \xi^{2}, P^{2}\right),
$$

where the corresponding $\mathcal{O}_{n}$ can be any two-currents operator defined in Eq. (2), and $\tilde{\omega} \equiv 2 P \cdot q /\left(-q^{2}\right)=1 / x_{B}$, with $x_{B}$ the Bjorken variable for the lepton-hadron deep inelastic scattering (DIS). Assuming $\left|P^{2} / q^{2}\right| \ll 1, \tilde{\sigma}_{n}$ has cuts going out to infinity from the thresholds $\tilde{\omega}= \pm 1$. The reason for having the cuts is that the system has a positive energy when $\tilde{\omega}^{2}>1$, corresponding to $(q+P)^{2}>0$ or $(q-P)^{2}>0$, and, thus, it can produce physically propagating particles. To understand the difference better, let us consider a simple integral which could contribute to Eq. (15),

$$
\int \frac{d^{4} \xi}{\xi^{4}} \xi^{\nu} e^{i(q+x P) \cdot \xi},
$$

where $-1<x<1$ could be thought as the momentum fraction of a parton inside of the hadron. If $q+x P$ is not lightlike for any value of $x$, which is equivalent to $\tilde{\omega}^{2}<1$, this integral is always finite. But if $q+x P$ is lightlike, this integral is divergent in the region where $\xi$ is large and almost anticollinear to $q+x P$. This simple exercise tells us that the nonanalytic cut of $\tilde{\sigma}_{n}$ comes from the integration region of large $\xi$. That is, even if we demand $\left|q^{2}\right| \gg \Lambda_{\mathrm{QCD}}^{2}, \tilde{\sigma}_{n}$ in momentum space can always receive contribution from large $\xi$ region so long as $\tilde{\omega}^{2}>1$. On the other hand, in coordinate space, if we fix $\xi$ to be a short distance, we do not have contribution from the large $\xi$ region and, thus, $\sigma_{n}$ has good analytic behavior.

Since $\tilde{\sigma}_{n}$ is simply a Fourier transformation of $\sigma_{n}$, the factorization of $\sigma_{n}$ in Eq. (8) implies the following factorization:

$$
\tilde{\sigma}_{n}=\sum_{a} f_{a} \otimes \tilde{K}_{n}^{a}+O\left(\Lambda_{\mathrm{QCD}}^{2} / q^{2}\right),
$$

where $\otimes$ represents the $x$ convolution in Eq. (8) and

$$
\tilde{K}_{n}^{a}=\int \frac{d^{4} \xi}{\xi^{4}} e^{i q \cdot \xi} K_{n}^{a}\left(x P \cdot \xi, \xi^{2}, x^{2} P^{2}, \mu\right) .
$$

From the discussion above, the factorization in momentum space is unambiguous if $\tilde{\omega}^{2}<1$.

Matching coefficients.-From Eq. (14), we can obtain $K_{n}^{a}$ if we know the Wilson coefficients $W_{n}^{(J, a)}$. Our strategy to calculate them is as follows: (i) calculate $K_{n}^{a}\left(x \omega, \xi^{2}, 0, \mu\right)$, which corresponds to the coefficient function in collinear factorization with $P^{2} \rightarrow 0$, (ii) expand $K_{n}^{a}\left(x \omega, \xi^{2}, 0, \mu\right)$ as a power series of $x \omega$, and (iii) compare it with $K_{n}^{a}\left(x \omega, \xi^{2}, 0, \mu\right)$ in Eq. (14), along with the fact $\Sigma_{J}(x \omega, 0)=(x \omega)^{J}$, to obtain $W_{n}^{(J, a)}$ as the expansion coefficients.

In the following, we calculate nonvanishing $K_{n}^{a}$ for various LCSs introduced above to the lowest order (LO) in $\alpha_{s}$ expansion, which we denote as $K_{n}^{a(0)}$. There is only one Feynman diagram shown in Fig. 1(a) that contributes to $K_{q}^{q(0)}$. According to our strategy above, we set $k^{\mu}=x P^{\mu}$ with $P^{2}=0$, and get

$$
K_{q}^{q(0)}\left(x \omega, \xi^{2}, 0, \mu\right)=\frac{1}{2} \operatorname{Tr}[\not k g] e^{i \xi \cdot k}=2 x \omega e^{i x \omega},
$$




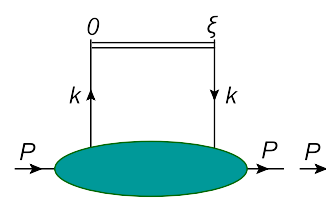

(a)

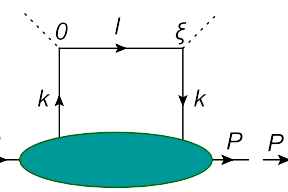

(b)

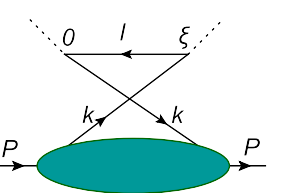

(c)
FIG. 1. LO Feynman diagrams for $\tilde{\sigma}_{n}$.

which is consistent with the relation Eq. (6). Two Feynman diagrams, as shown in Figs. 1(b) and 1(c), contribute to $K_{S}^{q(0)}$ with

$$
M_{b}=\frac{i \xi^{4}}{2} \int \frac{d^{4} l}{(2 \pi)^{4}} \frac{\operatorname{Tr}[\not k l] e^{i \xi \cdot(k-l)}}{l^{2}+i \varepsilon}=\frac{i}{\pi^{2}} x \omega e^{i x \omega},
$$

and $M_{c}=M_{b}^{*}$. We have the sum of these two diagrams,

$$
K_{S}^{q(0)}\left(x \omega, \xi^{2}, 0, \mu\right)=\frac{i}{\pi^{2}} x \omega\left(e^{i x \omega}-e^{-i x \omega}\right),
$$

which is consistent with the relation Eq. (4). Results of $K_{V}^{q(0)}$ and $K_{\tilde{V}}^{q(0)}$ are the same as $K_{S}^{q(0)}$ at this order. With $q \neq q^{\prime}$, only Fig. 1 (b) contributes to $K_{V^{\prime}}^{q(0)}$, while only Fig. 1(c) contributes to $K_{V^{\prime}}^{q^{\prime}(0)}$. Neglecting the mass of both $q$ and $q^{\prime}$, we obtain

$$
\begin{gathered}
K_{V^{\prime}}^{q(0)}\left(x \omega, \xi^{2}, 0, \mu\right)=\frac{i}{\pi^{2}} x \omega e^{i x \omega}, \\
K_{V^{\prime}}^{q^{\prime}(0)}\left(x \omega, \xi^{2}, 0, \mu\right)=\frac{-i}{\pi^{2}} x \omega e^{-i x \omega} .
\end{gathered}
$$

Using our strategy and the results of $K_{n}^{a(0)}$ evaluated at $P^{2}=0$ above, we can easily derive $W_{n}^{(J, a)}$. For example, by expanding $K_{q}^{q(0)}=\sum_{J=1} 2\left[i^{J-1} /(J-1) !\right](x \omega)^{J}$, and comparing it with $K_{q}^{q(0)}=\sum_{J} 2 W_{q}^{(J, q)}(x \omega)^{J}$ from Eq. (14) at $P^{2}=0$, we obtain $W_{q}^{(J, q)}=i^{J-1} /(J-1)$ ! with $J \geq 1$. Substituting this into Eq. (14), we obtain $K_{q}^{q(0)}\left(x \omega, \xi^{2}, x^{2} P^{2}, \mu\right)$. For simplifying our discussion below, we assume $\xi^{2}$ small enough so that $P^{2} \xi^{2}$ (and thus $P^{2}$ ) terms can be ignored in the rest of this Letter.

From Eq. (18), we can easily obtain $\tilde{K}_{n}^{a}$ using the above calculated $K_{n}^{a}$. For example, we have

$$
\tilde{K}_{S / V / \tilde{V}}^{q(0)}\left(x \tilde{\omega}, q^{2}, 0, \mu\right)=-2 i \frac{x^{2} \tilde{\omega}^{2}}{1-x^{2} \tilde{\omega}^{2}},
$$

which has cuts going out to infinity from the thresholds $\tilde{\omega}= \pm 1$ as discussed earlier.

Good lattice cross sections.-After showing that the UV renormalized coordinate-space hadronic matrix elements $\sigma_{n}$ in Eq. (1) can be factorized to the PDFs with perturbatively calculable coefficient functions, we need to demonstrate that these matrix elements are calculable in LQCD with Euclidean time, in order for them to be good lattice cross sections. This can be easily satisfied if $\xi$ has no time component. In conclusion, $\sigma_{n}$ defined in Eq. (1) are good LCSs for extracting PDFs under the condition $\xi_{0}=0$. For example, $\sigma_{S / V / \tilde{V}}$ can be naturally used to extract $f_{q}(x, \mu)+f_{\bar{q}}(x, \mu)$, and $\sigma_{V^{\prime}}$ can be used to extract $f_{q}(x, \mu)+f_{\bar{q}^{\prime}}(x, \mu)$. With various linear combinations of $\sigma_{n}$, we could extract $f_{q}(x, \mu), f_{\bar{q}}(x, \mu)$, and $f_{g}(x, \mu)$ individually.

If there are methods to calculate $\sigma_{n}$ or its linear combination in LQCD without setting $\xi_{0}=0$, then we can define more good LCSs accordingly. One possibility is that one can use the Feynman-Hellmann technique to calculate $\tilde{\sigma}_{n}$ with $q_{0}=0$ [29]. Therefore, according to our above discussion, $\tilde{\sigma}_{n}$ defined in Eq. (15) are also good LCSs for extracting PDFs under the condition $q_{0}=0$ and $\tilde{\omega}^{2}<1$.

Having many good LCSs makes it possible for extracting PDFs by using the QCD global analysis of "data" generated by LQCD calculations of these LCSs. This will provide a promising way to determine PDFs from $a b$ initio LQCD calculation. In fact, our method is so general that proposed LQCD calculable functions used in the literature to determine PDFs are special cases of these good LCSs, which we show in the following.

Relation to other methods.-Let us first discuss the relation to quasi-PDFs and pseudo-PDFs. With $K_{q}^{q(0)}$ calculated in Eq. (19), we can construct a linearly combined good LCS,

$$
\int \frac{d \omega}{\omega} \frac{e^{-i x \omega}}{4 \pi} \sigma_{q}\left(\omega, \xi^{2}, P^{2}\right) \approx f_{q}(x, \mu),
$$

modulo $O\left(\alpha_{s}\right)$ corrections and higher twist corrections. With $\xi_{0}=0$, the integral over $\omega=-\vec{\xi} \cdot \vec{P}=-|\vec{\xi}||\vec{P}| \cos \theta$ in Eq. (24) could have different interpretations. Choosing both $\vec{P}$ and $\vec{\xi}$ along the 3 direction, which results in $\theta=0$, the lefthand side of Eq. (24) is the quasiquark distribution introduced in Ref. [10] if the integration is performed by fixing $P_{3}$, while it is the pseudoquark distribution used in Ref. [29] if the integration is performed by fixing $\xi_{3}$. These two methods are equivalent if matching coefficients are calculated to lowest order in $\alpha_{s}$, but different if higher order contributions need to be considered. The Eq. (24) also tells us that, to effectively extract PDFs using good LCSs, one should generate lattice data with as many different values of $\omega$ as possible.

From Eq. (23) we have

$$
\tilde{\sigma}_{S / V / \tilde{V}} \approx-2 i \tilde{\omega} \int_{-1}^{1} d x \frac{x \tilde{\omega}}{1-x^{2} \tilde{\omega}^{2}} f_{q}\left(x, \mu^{2}\right),
$$

which is equivalent to $T_{33}$ used in Ref. [30] if we factorize the structure function therein to PDFs. More precisely, $T_{33}$ 
can be obtained if we construct momentum-space good LCSs $\tilde{\sigma}_{\mu \nu}$ using operator $\mathcal{O}_{\mu \nu}$ defined in Eq. (7), and then set $\mu=\nu=3$ and $P_{3}=q_{3}=q_{0}=0$. However, because $\tilde{\sigma}_{\mu \nu}$ has effectively only 2 degrees of freedom, all of its nontrivial information should have been encoded in $\tilde{\sigma}_{V}$ and $\tilde{\sigma}_{\tilde{V}}$. We thus expect that extracting PDFs using $\tilde{\sigma}_{V}$ and $\tilde{\sigma}_{\tilde{V}}$ can be at least as good as that using $T_{33}$.

Summary.-We constructed a class of good lattice cross sections in terms of single-hadron matrix elements of renormalized equal-time nonlocal operators in coordinate space, as defined in Eq. (1). We show that these matrix elements are calculable in LQCD and factorizable to PDFs with perturbative coefficients, so long as the largest separation between fields of the operator satisfies $|\vec{\xi}| \ll$ $1 / \Lambda_{\mathrm{QCD}}$. We also show that corresponding momentumspace matrix elements, $\tilde{\sigma}_{n}$ defined in Eq. (15) with $\mid 2 P$. $q /\left(-q^{2}\right) \mid<1$ and $\left|q^{2}\right| \gg \Lambda_{\mathrm{QCD}}^{2}$, are also good LCSs.

We provided some explicit examples of good LCSs made of quark fields in Eqs. (3) and (5), calculated corresponding matching coefficients to $\mathrm{LO}$ in $\alpha_{s}$, and connect the matrix elements to unpolarized PDFs. As briefly mentioned earlier, it is straightforward to construct the gluonic operators to have good LCSs directly sensitive to gluon distributions. Our approach could be easily applied for polarized PDFs and other parton correlation functions of various hadrons. We also find that some LQCD calculable functions used in literature for extracting PDFs, including quasi-PDFs proposed in Ref. [10], pseudo-PDFs used in Ref. [29], and $T_{33}$ used in Ref. [30], are special cases of good LCSs. With the large number of identified good LCSs, we could extract PDFs and hadron structure in general by QCD global analyses of the data generated by ab initio LQCD calculations of these LCSs.

Since it is very easy to generate lattice data with small $\omega$, LQCD calculations could provide the much needed information on PDFs at relatively large $x$, complementary to what could be extracted from experimental measurements. In addition, LQCD calculations could provide the information on partonic structure of hadrons, such as neutron, pion or keon, that could be difficult to do scattering experiments with.

We thank Chuan Liu, Xiaohui Liu, A. H. Mueller, and Feng Yuan for helpful communications. Especially, we thank Xu Feng for plenty of useful discussions. This work is supported in part by the U.S. Department of Energy, Office of Science, Office of Nuclear Physics under Award No. DE-AC05-06OR23177, within the framework of the TMD Topical Collaboration.

*yqma@pku.edu.cn jqiu@jlab.org

[1] Y.-Q. Ma and J.-W. Qiu, Extracting Parton Distribution Functions from Lattice QCD Calculations, arXiv:1404.6860.
[2] N. Brambilla et al., QCD and strongly coupled gauge theories: Challenges and perspectives, Eur. Phys. J. C 74, 2981 (2014).

[3] J. C. Collins, D. E. Soper, and G. Sterman, Factorization of hard processes in QCD, Adv. Ser. Dir. High Energy Phys. 5, 1 (1988).

[4] S. Dulat, T.-J. Hou, J. Gao, M. Guzzi, J. Huston, P. Nadolsky, J. Pumplin, C. Schmidt, D. Stump, and C. P. Yuan, New parton distribution functions from a global analysis of quantum chromodynamics, Phys. Rev. D 93, 033006 (2016).

[5] A. Martin, W. Stirling, R. Thorne, and G. Watt, Parton distributions for the LHC, Eur. Phys. J. C 63, 189 (2009).

[6] R. D. Ball et al. (NNPDF Collaboration), Parton distributions for the LHC Run II, J. High Energy Phys. 04 (2015) 040.

[7] S. Alekhin, J. Bluemlein, and S. Moch, The ABM parton distributions tuned to LHC data, Phys. Rev. D 89, 054028 (2014).

[8] J. J. Ethier, N. Sato, and W. Melnitchouk, First Simultaneous Extraction of Spin-Dependent Parton Distributions and Fragmentation Functions From A Global Qcd Analysis, Phys. Rev. Lett. 119, 132001 (2017).

[9] E. R. Nocera, H.-W. Lin, F. Olness, K. Orginos, and J. Rojo, The PDFLattice2017 Workshop: A Summary Report, 2017 arXiv:1709.01511.

[10] X. Ji, Parton Physics on a Euclidean Lattice, Phys. Rev. Lett. 110, 262002 (2013).

[11] H.-W. Lin, J.-W. Chen, S. D. Cohen, and X. Ji, Flavor structure of the nucleon sea from lattice QCD, Phys. Rev. D 91, 054510 (2015).

[12] C. Alexandrou, K. Cichy, V. Drach, E. Garcia-Ramos, K. Hadjiyiannakou, K. Jansen, F. Steffens, and C. Wiese, Lattice calculation of parton distributions, Phys. Rev. D 92, 014502 (2015).

[13] J.-W. Chen, S. D. Cohen, X. Ji, H.-W. Lin, and J.-H. Zhang, Nucleon helicity and transversity parton distributions from lattice QCD, Nucl. Phys. B911, 246 (2016).

[14] C. Alexandrou, K. Cichy, M. Constantinou, K. Hadjiyiannakou, K. Jansen, F. Steffens, and C. Wiese, Updated lattice results for parton distributions, Phys. Rev. D 96, 014513 (2017).

[15] J.-H. Zhang, J.-W. Chen, X. Ji, L. Jin, and H.-W. Lin, Pion distribution amplitude from lattice QCD, Phys. Rev. D 95, 094514 (2017).

[16] X. Ji, Parton physics from large-momentum effective field theory, Sci. China Phys. Mech. Astron. 57, 1407 (2014).

[17] X. Ji, J.-H. Zhang, and Y. Zhao, More on large-momentum effective theory approach to parton physics, Nucl. Phys. B924, 366 (2017).

[18] T. Ishikawa, Y.-Q. Ma, J.-W. Qiu, and S. Yoshida, Practical quasi parton distribution functions, arXiv:1609.02018.

[19] J.-W. Chen, X. Ji, and J.-H. Zhang, Improved quasi parton distribution through Wilson line renormalization, Nucl. Phys. B915, 1 (2017).

[20] C. Monahan and K. Orginos, Quasi parton distributions and the gradient flow, J. High Energy Phys. 03 (2017) 116.

[21] R. A. Briceño, M. T. Hansen, and C. J. Monahan, Role of the Euclidean signature in lattice calculations of 
quasidistributions and other nonlocal matrix elements, Phys. Rev. D 96, 014502 (2017).

[22] X. Xiong, T. Luu, and U.-G. Meißner, Quasi-Parton Distribution Function in Lattice Perturbation Theory, arXiv: 1705.00246.

[23] M. Constantinou and H. Panagopoulos, Perturbative renormalization of quasi-parton distribution functions, Phys. Rev. D 96, 054506 (2017).

[24] C. Alexandrou, K. Cichy, M. Constantinou, K. Hadjiyiannakou, K. Jansen, H. Panagopoulos, and F. Steffens, A complete non-perturbative renormalization prescription for quasi-PDFs, Nucl. Phys. B923, 394 (2017).

[25] J.-W. Chen, T. Ishikawa, L. Jin, H.-W. Lin, Y.-B. Yang, J.-H. Zhang, and Y. Zhao, Parton Distribution Function with Non-perturbative Renormalization from Lattice QCD, arXiv:1706.01295.

[26] X. Ji, J.-H. Zhang, and Y. Zhao, Renormalization in Large Momentum Effective Theory of Parton Physics, arXiv: 1706.08962.

[27] T. Ishikawa, Y.-Q. Ma, J.-W. Qiu, and S. Yoshida, On the renormalizability of quasi parton distribution functions, Phys. Rev. D 96, 094019 (2017).

[28] J. Green, K. Jansen, and F. Steffens, Nonperturbative renormalization of nonlocal quark bilinears for quasi-PDFs on the lattice using an auxiliary field, arXiv:1707.07152.

[29] K. Orginos, A. Radyushkin, J. Karpie, and S. Zafeiropoulos, Lattice QCD exploration of pseudo-PDFs, Phys. Rev. D 96, 094503 (2017).
[30] A. J. Chambers, R. Horsley, Y. Nakamura, H. Perlt, P. E. L. Rakow, G. Schierholz, A. Schiller, K. Somfleth, R. D. Young, and J. M. Zanotti, Nucleon Structure Functions from Operator Product Expansion on the Lattice, Phys. Rev. Lett. 118, 242001 (2017).

[31] K.-F. Liu and S.-J. Dong, Origin of Difference Between $\bar{u}$ and $\bar{d}$ Partons in the Nucleon, Phys. Rev. Lett. 72, 1790 (1994).

[32] K.-F. Liu, Parton degrees of freedom from the path integral formalism, Phys. Rev. D 62, 074501 (2000).

[33] K.-F. Liu, Parton Distribution Function from the Hadronic Tensor on the Lattice, Proc. Sci., LATTICE2015 (2016) 115.

[34] U. Aglietti, M. Ciuchini, G. Corbo, E. Franco, G. Martinelli, and L. Silvestrini, Model independent determination of the light cone wave functions for exclusive processes, Phys. Lett. B 441, 371 (1998).

[35] A. Abada, P. Boucaud, G. Herdoiza, J. P. Leroy, J. Micheli, O. Pene, and J. Rodriguez-Quintero, Preliminaries on a lattice analysis of the pion light cone wave function: A Partonic signal?, Phys. Rev. D 64, 074511 (2001).

[36] V. Braun and D. Mueller, Exclusive processes in position space and the pion distribution amplitude, Eur. Phys. J. C 55, 349 (2008).

[37] J. C. Collins, Renormalization. An Introduction To Renormalization, The Renormalization Group, and the Operator Product Expansion, Cambridge Monographs on Mathematical Physics Vol. 26 (Cambridge University Press, Cambridge, England, 1986). 